\title{
The HOTAIR, PRNCR1 and POLR2E polymorphisms are associated with cancer risk: a meta-analysis
}

\author{
Haiyan Chu ${ }^{1,2,3}$, Yaoyao Chen ${ }^{1}$, Qinbo Yuan ${ }^{4,5}$, Qiuhan Hua ${ }^{1}$, Xu Zhang ${ }^{1}$, Meilin \\ Wang $^{1,2}$, Na Tong ${ }^{1}$, Wei Zhang ${ }^{5}$, Jinfei $\mathrm{Chen}^{3}$ and Zhengdong Zhang ${ }^{1,2}$ \\ ${ }^{1}$ Department of Genetic Toxicology, The Key Laboratory of Modern Toxicology of Ministry of Education, School of Public \\ Health, Nanjing Medical University, Nanjing, China \\ ${ }^{2}$ Department of Environmental Genomics, Jiangsu Key Laboratory of Cancer Biomarkers, Prevention and Treatment, \\ Collaborative Innovation Center for Cancer Personalized Medicine, Nanjing Medical University, Nanjing, China \\ ${ }^{3}$ Department of Oncology, The Affiliated Nanjing First Hospital, Nanjing Medical University, Nanjing, China \\ ${ }^{4}$ Department of Urology, Huaiyin Hospital of Huai'an City, Huai'an, China \\ ${ }^{5}$ Department of Urology, The First Affiliated Hospital of Nanjing Medical University, Nanjing, China \\ Correspondence to: Zhengdong Zhang, email: drzdzhang@gmail.com
}

Keywords: IncRNA, polymorphism, meta-analysis, cancer, epidemiology

Received: August 29, $2016 \quad$ Accepted: December 27, $2016 \quad$ Published: January 31, 2017

Copyright: Chu et al. This is an open-access article distributed under the terms of the Creative Commons Attribution License 3.0 (CC BY 3.0), which permits unrestricted use, distribution, and reproduction in any medium, provided the original author and source are credited.

\section{ABSTRACT}

Long non-coding RNAs (LncRNAs) have been widely studied and aberrant expression of IncRNAs are involved in diverse cancers. Genetic variation in IncRNAs can influence the IncRNAs expression and function. At present, there are many studies to investigate the association between IncRNAs polymorphisms and cancer susceptibility. However, it has no systematic study to evaluate the association. We performed a metaanalysis to summarize the results of common IncRNAs (HOTAIR, PRNCR1, POLR2E and $H 19$ ) polymorphisms on cancer risk, by using the random-effect model to obtain the odds ratio (ORs) and $95 \%$ confidence interval $(95 \% \mathrm{CI})$. We also applied the meta-regression and publication bias analysis to seek the source of heterogeneity and evaluate the stability of results, respectively. The summary results indicated that HOTAIR rs920778 increased the cancer risk in recessive model (OR $=1.61,95 \% \mathrm{CI}$ $=1.08-2.41, P_{\text {heterogeneity }}<0.001$ ). For PRNCR1 (rs1016343, rs16901946) and POLR2E (rs3787016), we also found the significant association with incresed risk of cancer (all $P<0.05)$. However, we did not observe any significant association between $H 19$ rs2107425 and cancer risk. Our meta-analysis results revealed that these four IncRNAs polymorphisms (HOTAIR rs920778, PRNCR1 rs1016343 and rs16901946, POLR2E rs3787016) can contribute to cancer risk. Further studies should confirm these findings.

\section{INTRODUCTION}

Long non-coding RNAs (LncRNAs) are a class of $>200 \mathrm{nt}$ in length non-coding RNAs, which are involved in diverse cellular processes, such as cell cycle, apoptosis, epigenetics, and gene expression regulation $[1,2]$. LncRNAs have several subtypes mainly according to the relationship between lncRNAs' location and corresponding protein-coding gene's location, including sense lncRNAs, antisense lncRNAs, intergenic lncRNAs, intronic lncRNAs and bidirectional lncRNAs [3, 4]. With the development of high throughput chip and sequencing technology, a variety of lncRNAs have been investigated, e.g. HOX transcript antisense RNA (HOTAIR) [5], prostate cancer non-coding RNA1 (PRNCR1) [6], RNA polymerase II polypeptise E gene (POLR2E) [7, 8].

Emerging studies had shown that abnormal expression of IncRNAs was associated with cancer risk. Schmidt and the colleagues reported that the overexpression non-coding metastasis associated lung adenocarcinoma transcript 1 (MALAT1) can increase the lung cancer cell proliferation and migration activity [9]. Similarly, increased expression of HOTAIR contributes to the breast cancer poor prognosis and metastasis [10]. 
In our previous study, we also identified the antisense of mediator of DNA damage checkpoint protein 1 (MDC1) acting as the suppressor gene involved in bladder cancer [11]. However, the exact etiology is still not clear.

Single nucleotide polymorphisms (SNPs) can influence the gene expression and function, participating in carcinogenesis [12]. Recently, SNPs in HOTAIR have been widely studied in multiple cancers [13-21]. Yan et al. performed a population-based study to investigate the association between HOTAIR polymorphisms (rs1899663, rs4759314, rs920778) and breast cancer risk, and result indicated that HOTAIR rs920778 was associated with the increased risk of breast cancer [14], similarly in esophageal cancer [20]. We also observed that HOTAIR rs7958904 can decrease colorectal cancer risk [21]. Besides, we found that IncRNA PRNCR1 also have been widely investigated in the development of cancer, and PRNCR1 firstly identified and named in prostate cancer [6, 22-26]. For PRNCR1, rs1016343 had been studied widely. It had been reported that Salinas et al. investigated the association between PRNCR1 rs1016343 and prostate cancer risk in Caucasians and African Americans; however, they only found rs1016343 associated with increased risk of prostate cancer in Caucasians, and no significant association was observed in African Americans [6]. In gastric cancer, Li et al. did not find the significant risk for rs1016343, and find that PRNCR1 rs13252298, rs7007694, rs1456315 polymorphisms were associated with gastric cancer risk [22]. Additionally, lncRNA H19
[27-32] and POLR2E [7, 8, 33, 34] polymorphisms were studied widely. The published results of $H 19$ and POLR2E polymorphisms with cancer risk were also conflicted. In summary, we noticed that the results of IncRNAs publications were conflicted rather than conclusive. Herein, we conducted a meta-analysis to summarize all eligible case-control studies to evaluate the overall cancer risk and common IncRNAs (HOTAIR, PRNCR1, H19, and POLR2E) polymorphisms.

\section{RESULTS}

\section{Characteristics of included studies}

Through searching the PubMed using the key words, we retrieved 344 relevant articles (Figure 1). In addition, we also retrieved 22 articles by manual search of the references of relevant articles. After reviewing the title or abstract, we excluded 283 unrelated articles, leaving 83 articles for further evaluation. We carefully evaluated the full text of articles, and excluded 45 articles (27, not case-control study; 14, no detailed genotype frequency; four, not human cancer study). In these 38 included articles, we observed that a variety of lncRNA genes had been investigated the association with cancer risk, among which lncRNA HOTAIR, PRNCR1, H19, and POLR2E had been widely studied. Thus, in the meta-analysis, we mainly focus on evaluating the association between HOTAIR, PRNCR1, H19, POLR2E polymorphisms and

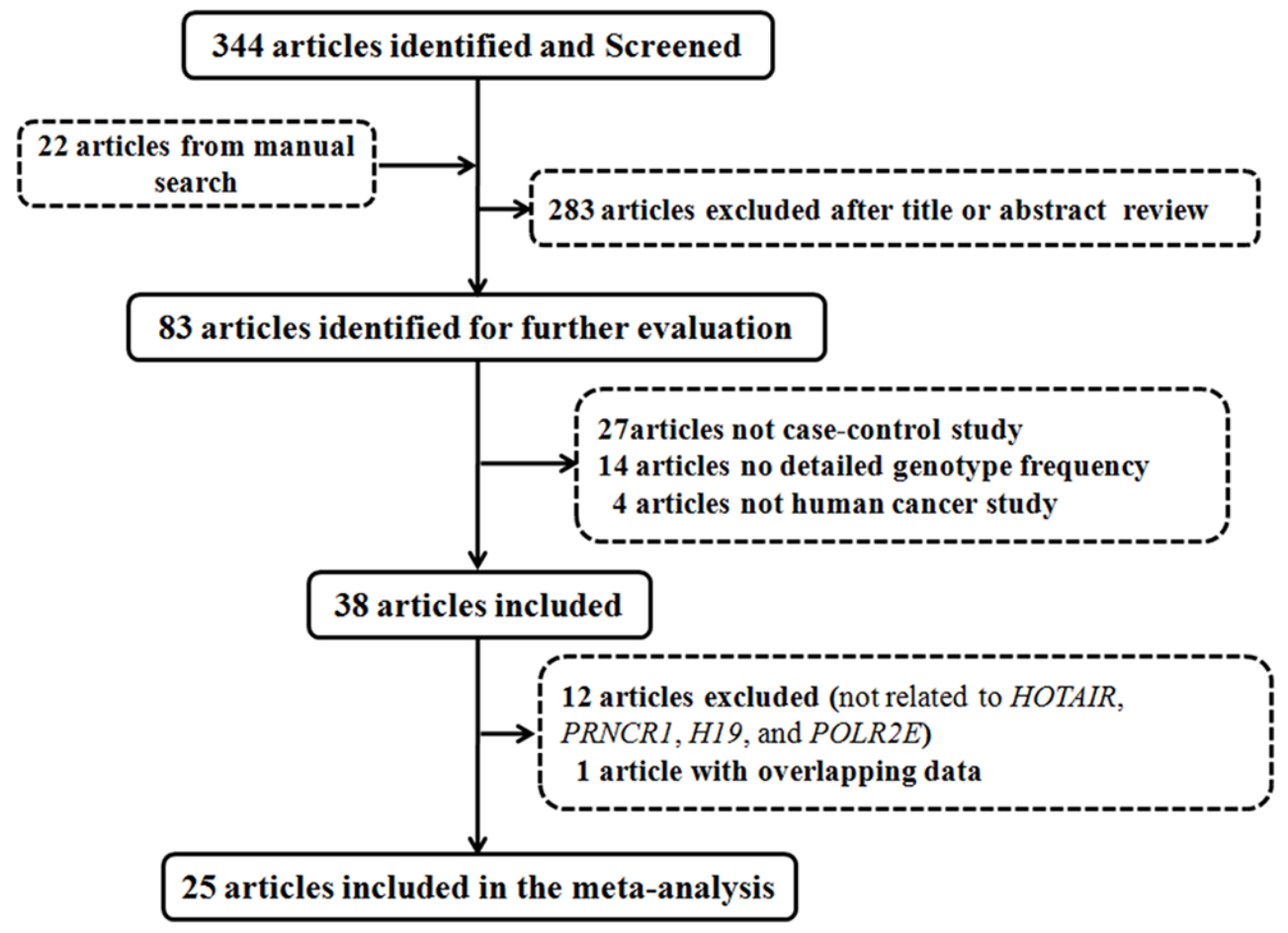

Figure 1: Flow diagram of articles identified with included and excluded criteria. 
cancer risk. Finally, we included 25 relevant articles in this meta-analysis (nine for HOTAIR, six for PRNCR1, six for H19, and four for POLR2E).

As shown in Supplementary Table 1, we summarized the characteristics of the included studies. For HOTAIR, there were six studies from China (Asian) and three studies from Turkey (Caucasian), and the source of control of studies mainly was hospital-based population (eight studies). For PRNCR1, we also observed that major studies were from Chinese population (67\%), and four studies were involved in prostate cancer. For POLR2E, there were three studies about prostate cancer, one about esophageal cancer. While for H19, studies were including two breast cancer studies, one gastric cancer study, one melanoma study, one ovarian cancer study, and one bladder cancer study. The majority of these studies were matched with age and sex. We found that these included studies mainly used polymerase chain reaction-restriction fragment length polymorphism (PCR-RFLP) assay (8 studies) and TaqMan assay ( 9 studies) to genotype the polymorphisms. In this meta-analysis, all included SNP genotypes frequency in control was consistent with HWE.

\section{Quantitative synthesis}

We found that the HOTAIR, PRNCR1, H19, $P O L R 2 E$ genes were presented very polymorphic, however, not all polymorphic loci had been widely studied. We summarized the common SNPs in Table 1 (the numbers of SNPs $>3$ ). For HOTAIR, we noticed that there were six studies about rs4759314, five studies about rs920778, and three studies about rs1899663. Herein, we assessed the association between these three SNPs (rs4759314, 920778, and rs1899663) and cancer risk (Table 2). Meta-analysis result showed that rs920778 increased the cancer risk in recessive model $(\mathrm{OR}=1.61$, $\left.95 \% \mathrm{CI}=1.08-2.41, P_{\text {heterogeneity }}<0.001\right)$ and additive model (1.24, 1.03-1.49, 0.001). For PRNCR1, studies mainly reported the relationship of $\operatorname{rs} 1016343(\mathrm{n}=7), \operatorname{rs} 13252298$ $(\mathrm{n}=4), \mathrm{rs} 7007694(\mathrm{n}=3), \mathrm{rs} 16901946(\mathrm{n}=3), \mathrm{rs} 1456315$ $(\mathrm{n}=3)$ and cancer risk (Table 2). We further evaluated the association between these SNPs and cancer risk, and found that both rs1016343 and rs16901946 increased the risk of cancer in the dominant model $(1.27,1.04$ $1.53,0.002 ; 1.15 .1 .02-1.29,0.573$, respectively). In the additive model, we also observed that rs 1016343 was associated with a $24 \%$ increased risk of cancer (1.24, $1.04-1.47,<0.001)$. For H19, several studies investigated the association between SNPs and cancer risk, among which there were four studies about rs2107425 (Table 1). We then performed a meta-analysis to evaluate the association between rs2107425 and cancer risk (Table 2). Overall, we observed that rs2107425 could decrease the risk of cancer with the borderline effect in the dominat model $(0.89,0.80-0.99,0.166)$, and no significant effect was found in other models (recessive model: 1.04, 0.94-
1.16, 0.651; additive model: 0.92, 0.84-1.02, 0.173). For POLR2E, only one SNP rs3787016 had been investigated the association with cancer risk in the case-control studies. Meta-analysis result suggested the individuals with rs3787016 TT genotype had a 1.52-fold significantly increased cancer risk in the recessive model $(1.52,1.17$ 1.97, 0.201).

Further, we evaluated the effects of lncRNA SNPs according to ethnicity and cancer type. We mainly focused on investigating the effects of three lncRNA polymorphisms (HOTAIR rs920778, PRNCR1 rs1016343, and POLR2E rs3787016) in subgroups. As shown in Table 3, HOTAIR rs920778 contributed to the increased risk of esophageal cancer in all models (dominant model: 1.48, 1.31-1.67; recessive model: 2.51, 1.91-3.29; additive model: 1.48, 1.34-1.64). We also observed the similar effect in Asians (dominant model: 1.46, 1.33-1.61, 0.953; recessive model: 2.13, 1.42-3.20, 0.004; additive model: 1.46, 1.35-1.57, 0.803). For PRNCR1 rs1016343, in stratified analyses by cancer type, increased risks were observed for prostate cancer in all models (dominant model: $1.39,1.12-1.71,0.012$; recessive model: $1.83,1.53$ $2.18,0.855$; additive model: $1.42,1.29-1.56,0.257)$. The similar associations were found in Caucasian populations (dominant model: 1.39, 1.18-1.63; recessive model: 1.72, 1.21-2.44; additive model: 1.36, 1.19-1.55). For POLR2E, we found that rs3787016 increased the risk of esophageal cancer (recessive model: 1.76, 1.25-2.49). In addition, elevated risk also was observed in Asian populations (recessive model: 1.64, 1.35-1.99, 0.624).

\section{Meta-regression}

We observed the significant heterogeneity between studies in the meta-analysis. Then, we performed the metaregression analysis to assess the source of heterogeneity in the additive model (Table 4). For HOTAIR rs920778, we observed that ethnicity and genotyping method were the source of heterogeneity (all $P<0.001$ ), and could explain $100 \%$ of the Tau-squared. Similarly, we also found that the source of control $(P=0.002)$ and genotyping method $(P=0.047)$ could explain the $100 \%$ of the Tau-squared for the POLR2E rs3787016. Additionally, for the PRNCR1 rs1016343, cancer type $(P<0.001)$ was observed to contribute to substantial heterogeneity $(95 \%$ of the Tausquared).

\section{Publication bias}

Egger's test and Begg's test were used to evaluate the publication bias of the HOTAIR rs920778, PRNCR1 rs1016343, POLR2E rs3787016 in the meta-analysis. Egger's test results demonstrated that there was a significant publication bias in the additive model for the HOTAIR rs920778 $(t=-3.91, P=0.030)$, and no significant publication bias was found for the other two 
Table 1: Characteristics of the included studies

\begin{tabular}{|c|c|c|c|c|c|c|c|c|c|c|}
\hline Gene & Authors & Year & Country & Ethnicity & $\begin{array}{c}\text { Source } \\
\text { of } \\
\text { control }\end{array}$ & SNP & $\begin{array}{c}\text { Cancer } \\
\text { type }\end{array}$ & $\begin{array}{c}\text { Genotyping } \\
\text { method }\end{array}$ & Case & Control \\
\hline \multirow[t]{14}{*}{ HOTAIR } & Yan et al. & 2015 & China & Asian & PB & $\begin{array}{c}\mathrm{rs} 1899663 \\
\mathrm{G}>\mathrm{T}\end{array}$ & $\begin{array}{l}\text { Breast } \\
\text { cancer }\end{array}$ & PCR-RFLP & 502 & 504 \\
\hline & & & & & & $\begin{array}{c}\text { rs4759314 } \\
A>G\end{array}$ & & & 502 & 504 \\
\hline & & & & & & $\begin{array}{l}\text { rs920778 } \\
\mathrm{C}>\mathrm{T}\end{array}$ & & & 502 & 504 \\
\hline & Du et al. & 2015 & China & Asian & HB & $\begin{array}{c}\text { rs4759314 } \\
A>G\end{array}$ & $\begin{array}{l}\text { Gastric } \\
\text { cancer }\end{array}$ & TaqMan & 1275 & 1644 \\
\hline & Pan et al. & 2015 & China & Asian & HB & $\begin{array}{c}\mathrm{rs} 1899663 \\
\mathrm{G}>\mathrm{T}\end{array}$ & $\begin{array}{l}\text { Gastric } \\
\text { cancer }\end{array}$ & PCR-RFLP & 500 & 1000 \\
\hline & & & & & & $\begin{array}{c}\mathrm{rs} 4759314 \\
\mathrm{~A}>\mathrm{G}\end{array}$ & & & 500 & 1000 \\
\hline & & & & & & $\begin{array}{c}\text { rs920778 } \\
\mathrm{C}>\mathrm{T}\end{array}$ & & & 800 & 1600 \\
\hline & Guo et al. & 2015 & China & Asian & HB & $\begin{array}{c}\mathrm{rs} 4759314 \\
\mathrm{~A}>\mathrm{G}\end{array}$ & $\begin{array}{l}\text { Gastric } \\
\text { cancer }\end{array}$ & PCR-RFLP & 515 & 654 \\
\hline & Bayram et al. & 2015 & Turky & Caucasian & HB & $\begin{array}{c}\text { rs920778 } \\
\mathrm{C}>\mathrm{T}\end{array}$ & $\begin{array}{l}\text { Gastric } \\
\text { cancer }\end{array}$ & TaqMan & 104 & 209 \\
\hline & Bayram et al. & 2015 & Turky & Caucasian & HB & $\begin{array}{c}\text { rs920778 } \\
\mathrm{C}>\mathrm{T}\end{array}$ & $\begin{array}{l}\text { Breast } \\
\text { cancer }\end{array}$ & TaqMan & 123 & 122 \\
\hline & Xue et al. & 2014 & China & Asian & HB & $\begin{array}{c}\mathrm{rs} 4759314 \\
\mathrm{~A}>\mathrm{G}\end{array}$ & $\begin{array}{c}\text { Colorectal } \\
\text { cancer }\end{array}$ & TaqMan & 1733 & 1855 \\
\hline & Zhang et al. & 2014 & China & Asian & HB & $\begin{array}{c}\text { rs } 1899663 \\
\mathrm{G}>\mathrm{T}\end{array}$ & $\begin{array}{c}\text { Esophageal } \\
\text { cancer }\end{array}$ & PCR-RFLP & 1000 & 1000 \\
\hline & & & & & & $\begin{array}{c}\mathrm{rs} 4759314 \\
\mathrm{~A}>\mathrm{G}\end{array}$ & & & 1000 & 1000 \\
\hline & & & & & & $\begin{array}{c}\text { rs920778 } \\
\mathrm{C}>\mathrm{T}\end{array}$ & & & 2098 & 2150 \\
\hline \multirow[t]{7}{*}{ PRNCR1 } & Li et al. & 2015 & China & Asian & HB & $\begin{array}{c}\mathrm{rs} 1016343 \\
\mathrm{C}>\mathrm{T}\end{array}$ & $\begin{array}{l}\text { Gastric } \\
\text { cancer }\end{array}$ & PCR-RFLP & 219 & 394 \\
\hline & & & & & & $\begin{array}{c}\mathrm{rs} 13252298 \\
\mathrm{~A}>\mathrm{G}\end{array}$ & & & 219 & 394 \\
\hline & & & & & & $\begin{array}{c}\text { rs } 7007694 \\
\mathrm{~T}>\mathrm{C}\end{array}$ & & & 219 & 394 \\
\hline & & & & & & $\begin{array}{c}\mathrm{rs} 16901946 \\
\mathrm{~A}>\mathrm{G}\end{array}$ & & & 219 & 394 \\
\hline & & & & & & $\begin{array}{c}\mathrm{rs} 1456315 \\
\mathrm{~A}>\mathrm{G}\end{array}$ & & & 219 & 394 \\
\hline & Hui et al. & 2014 & China & Asian & HB & $\begin{array}{c}\mathrm{rs} 1016343 \\
\mathrm{C}>\mathrm{T}\end{array}$ & $\begin{array}{l}\text { Prostate } \\
\text { cancer }\end{array}$ & PCR-HRM & 284 & 284 \\
\hline & & & & & & $\begin{array}{c}\mathrm{rs} 13252298 \\
\mathrm{~A}>\mathrm{G}\end{array}$ & & & 277 & 267 \\
\hline
\end{tabular}

(Continued) 


\begin{tabular}{|c|c|c|c|c|c|c|c|c|c|c|}
\hline Gene & Authors & Year & Country & Ethnicity & $\begin{array}{c}\text { Source } \\
\text { of } \\
\text { control }\end{array}$ & SNP & $\begin{array}{c}\text { Cancer } \\
\text { type }\end{array}$ & $\begin{array}{l}\text { Genotyping } \\
\text { method }\end{array}$ & Case & Control \\
\hline & Li et al. & 2013 & China & Asian & $\mathrm{HB}$ & $\begin{array}{c}\mathrm{rs} 1016343 \\
\mathrm{C}>\mathrm{T}\end{array}$ & $\begin{array}{c}\text { Colorectal } \\
\text { cancer }\end{array}$ & PCR-RFLP & 313 & 595 \\
\hline & \multirow{9}{*}{ Chung et al. } & \multirow{9}{*}{2011} & \multirow{9}{*}{ Japan } & \multirow{9}{*}{ Asian } & \multirow{9}{*}{$\mathrm{HB}$} & $\begin{array}{c}\mathrm{rs} 13252298 \\
\mathrm{~A}>\mathrm{G}\end{array}$ & & & 313 & 595 \\
\hline & & & & & & $\begin{array}{c}\mathrm{rs} 7007694 \\
\mathrm{~T}>\mathrm{C}\end{array}$ & & & 313 & 595 \\
\hline & & & & & & $\begin{array}{c}\mathrm{rs} 16901946 \\
\mathrm{~A}>\mathrm{G}\end{array}$ & & & 313 & 595 \\
\hline & & & & & & $\begin{array}{c}\mathrm{rs} 1456315 \\
\mathrm{~A}>\mathrm{G}\end{array}$ & & & 313 & 595 \\
\hline & & & & & & $\begin{array}{c}\mathrm{rs} 1016343 \\
\mathrm{C}>\mathrm{T}\end{array}$ & $\begin{array}{c}\text { Prostate } \\
\text { cancer }\end{array}$ & $\begin{array}{c}\text { Multiplex } \\
\text { PCR-based }\end{array}$ & 1502 & 1552 \\
\hline & & & & & & $\begin{array}{c}\text { rs } 13252298 \\
A>G\end{array}$ & & Invader assay & 1501 & 1550 \\
\hline & & & & & & $\begin{array}{c}\mathrm{rs} 7007694 \\
\mathrm{~T}>\mathrm{C}\end{array}$ & & & 1497 & 1554 \\
\hline & & & & & & $\begin{array}{c}\mathrm{rs} 16901946 \\
\mathrm{~A}>\mathrm{G}\end{array}$ & & & 1504 & 1554 \\
\hline & & & & & & $\begin{array}{c}\mathrm{rs} 1456315 \\
\mathrm{~A}>\mathrm{G}\end{array}$ & & & 1504 & 1553 \\
\hline & Zheng et al. & 2010 & China & Asian & HB & $\begin{array}{c}\mathrm{rs} 1016343 \\
\mathrm{C}>\mathrm{T}\end{array}$ & $\begin{array}{l}\text { Prostate } \\
\text { cancer }\end{array}$ & $\begin{array}{c}\text { MassARRAY } \\
\text { iPLEX } \\
\text { system }\end{array}$ & 284 & 147 \\
\hline & \multirow[t]{2}{*}{ Salinas et al. } & \multirow[t]{2}{*}{2008} & \multirow[t]{2}{*}{ USA } & Caucasian & PB & $\begin{array}{c}\mathrm{rs} 1016343 \\
\mathrm{C}>\mathrm{T}\end{array}$ & $\begin{array}{l}\text { Prostate } \\
\text { cancer }\end{array}$ & SNPlex & 1253 & 1233 \\
\hline & & & & $\begin{array}{c}\text { African } \\
\text { Americans }\end{array}$ & PB & $\begin{array}{c}\mathrm{rs} 1016343 \\
\mathrm{C}>\mathrm{T}\end{array}$ & $\begin{array}{l}\text { Prostate } \\
\text { cancer }\end{array}$ & SNPlex & 143 & 79 \\
\hline \multirow[t]{4}{*}{ H19 } & Butt et al. & 2012 & Sweden & Caucasian & PB & $\begin{array}{c}\mathrm{rs} 2107425 \\
\mathrm{C}>\mathrm{T}\end{array}$ & $\begin{array}{l}\text { Breast } \\
\text { cancer }\end{array}$ & $\begin{array}{c}\text { MALDI-TOF } \\
\text { MS }\end{array}$ & 679 & 1355 \\
\hline & Song et al. & 2009 & UK & Caucasian & PB & $\begin{array}{c}\mathrm{rs} 2107425 \\
\mathrm{C}>\mathrm{T}\end{array}$ & $\begin{array}{l}\text { Ovarian } \\
\text { cancer }\end{array}$ & TaqMan & 5366 & 8538 \\
\hline & Verhaegh et al. & 2008 & Netherlands & Caucasian & PB & $\begin{array}{c}\mathrm{rs} 2107425 \\
\mathrm{C}>\mathrm{T}\end{array}$ & $\begin{array}{l}\text { Bladder } \\
\text { cancer }\end{array}$ & PCR-RFLP & 177 & 204 \\
\hline & Bhatti et al. & 2008 & USA & Mixed & $\mathrm{PB}$ & $\begin{array}{c}\mathrm{rs} 2107425 \\
\mathrm{C}>\mathrm{T}\end{array}$ & $\begin{array}{l}\text { Breast } \\
\text { cancer }\end{array}$ & Unknown & 824 & 1073 \\
\hline \multirow[t]{4}{*}{ POLR2E } & Kang et al. & 2015 & China & Asian & HB & $\begin{array}{c}\text { rs3787016 } \\
\mathrm{C}>\mathrm{T}\end{array}$ & $\begin{array}{c}\text { Esophageal } \\
\text { cancer }\end{array}$ & $\begin{array}{c}\text { MALDI-TOF } \\
\text { MS }\end{array}$ & 369 & 370 \\
\hline & Cao et al. & 2014 & China & Asian & PB & $\begin{array}{c}\mathrm{rs} 3787016 \\
\mathrm{C}>\mathrm{T}\end{array}$ & $\begin{array}{l}\text { Prostate } \\
\text { cancer }\end{array}$ & TaqMan & 1015 & 1032 \\
\hline & Nicolic et al. & 2013 & Serbia & Caucasian & $\mathrm{HB}$ & $\begin{array}{c}\mathrm{rs} 3787016 \\
\mathrm{C}>\mathrm{T}\end{array}$ & $\begin{array}{l}\text { Prostate } \\
\text { cancer }\end{array}$ & TaqMan & 261 & 293 \\
\hline & Jin et al. & 2011 & USA & Caucasian & PB & $\begin{array}{c}\text { rs3787016 } \\
\mathrm{C}>\mathrm{T}\end{array}$ & $\begin{array}{c}\text { Prostate } \\
\text { cancer }\end{array}$ & $\begin{array}{l}\text { Illumina chip, } \\
\text { MassARRAY }\end{array}$ & 4196 & 5007 \\
\hline
\end{tabular}

PB: population based; HB: hospital based; PCR-RFLP: polymerase chain reaction restriction fragment length polymorphism; MALDI-TOF MS: matrix-assisted laser desorption/ionization time-of-flight mass spectrometry 
Table 2: Pooled analyses of IncRNA polymorphims on cancer risk

\begin{tabular}{|c|c|c|c|c|c|c|c|c|c|c|c|}
\hline \multirow[b]{2}{*}{ Gene } & \multirow[b]{2}{*}{ SNPs } & \multirow{2}{*}{$\begin{array}{c}\text { Allele } \\
\text { (major/ } \\
\text { minor) }\end{array}$} & \multirow[b]{2}{*}{$\mathbf{n}^{\mathbf{a}}$} & \multirow[b]{2}{*}{ Cases } & \multirow[b]{2}{*}{ Controls } & \multicolumn{2}{|c|}{ Dom model } & \multicolumn{2}{|c|}{ Rec model } & \multicolumn{2}{|c|}{ Add model } \\
\hline & & & & & & $\begin{array}{c}\text { OR } \\
(95 \% \mathrm{CI})\end{array}$ & $P^{\mathbf{b}}$ & $\begin{array}{c}\text { OR }(95 \% \\
\text { CI })\end{array}$ & $P^{\mathbf{b}}$ & $\begin{array}{c}\text { OR } \\
(95 \% \mathrm{CI})\end{array}$ & $P^{\mathbf{b}}$ \\
\hline \multirow[t]{3}{*}{ HOTAIR } & rs1899663 & $\mathrm{G}>\mathrm{T}$ & 3 & 2002 & 2504 & $\begin{array}{c}0.94 \\
(0.82-1.07)\end{array}$ & 0.714 & $\begin{array}{c}0.75 \\
(0.49-1.12)\end{array}$ & 0.891 & $\begin{array}{c}0.93 \\
(0.83-1.04)\end{array}$ & 0.774 \\
\hline & rs4759314 & $\mathrm{A}>\mathrm{G}$ & 6 & 5525 & 6657 & $\begin{array}{c}1.05 \\
(0.86-1.28)\end{array}$ & 0.024 & $\begin{array}{c}0.75 \\
(0.39-1.41)\end{array}$ & 0.880 & $\begin{array}{c}1.04 \\
(0.86-1.25)\end{array}$ & 0.027 \\
\hline & rs920778 & $\mathrm{C}>\mathrm{T}$ & 5 & 3627 & 4585 & $\begin{array}{c}1.20 \\
(0.92-1.57)\end{array}$ & 0.005 & $\begin{array}{c}1.61 \\
(1.08-2.41)\end{array}$ & $<0.001$ & $\begin{array}{c}1.24 \\
(1.03-1.49)\end{array}$ & 0.001 \\
\hline \multirow[t]{5}{*}{ PRNCR1 } & rs 1016343 & $\mathrm{C}>\mathrm{T}$ & 7 & 3998 & 4284 & $\begin{array}{c}1.27 \\
(1.04-1.53)\end{array}$ & 0.002 & $\begin{array}{c}1.33 \\
(0.92-1.92)\end{array}$ & $<0.001$ & $\begin{array}{c}1.24 \\
(1.04-1.47)\end{array}$ & $<0.001$ \\
\hline & rs 13252298 & $\mathrm{~A}>\mathrm{G}$ & 4 & 2310 & 2806 & $\begin{array}{c}0.84 \\
(0.55-1.28)\end{array}$ & $<0.001$ & $\begin{array}{c}0.89 \\
(0.63-1.27)\end{array}$ & 0.059 & $\begin{array}{c}0.89 \\
(0.65-1.22)\end{array}$ & $<0.001$ \\
\hline & rs7007694 & $\mathrm{T}>\mathrm{C}$ & 3 & 2029 & 2543 & $\begin{array}{c}0.93 \\
(0.71-1.22)\end{array}$ & 0.027 & $\begin{array}{c}1.10 \\
(0.63-1.89)\end{array}$ & 0.053 & $\begin{array}{c}0.96 \\
(0.74-1.25)\end{array}$ & 0.008 \\
\hline & rs16901946 & $\mathrm{A}>\mathrm{G}$ & 3 & 2036 & 2543 & $\begin{array}{c}1.15 \\
(1.02-1.29)\end{array}$ & 0.573 & $\begin{array}{c}0.92 \\
(0.37-2.26)\end{array}$ & 0.002 & $\begin{array}{c}1.09 \\
(0.91-1.30)\end{array}$ & 0.080 \\
\hline & rs 1456315 & $\mathrm{~A}>\mathrm{G}$ & 3 & 2036 & 2542 & $\begin{array}{c}0.70 \\
(0.46-1.05)\end{array}$ & $<0.001$ & $\begin{array}{c}0.64 \\
(0.31-1.31)\end{array}$ & 0.002 & $\begin{array}{c}0.74 \\
(0.53-1.04)\end{array}$ & $<0.001$ \\
\hline H19 & rs2107425 & $\mathrm{C}>\mathrm{T}$ & 4 & 7046 & 11170 & $\begin{array}{c}0.89 \\
(0.80-0.99)\end{array}$ & 0.166 & $\begin{array}{c}1.04 \\
(0.94-1.16)\end{array}$ & 0.651 & $\begin{array}{c}0.92 \\
(0.84-1.02)\end{array}$ & 0.173 \\
\hline POLR2E & rs3787016 & $\mathrm{C}>\mathrm{T}$ & 4 & 5841 & 6702 & $\begin{array}{c}0.87 \\
(0.61-1.26)\end{array}$ & 0.005 & $\begin{array}{c}1.52 \\
(1.17-1.97)\end{array}$ & 0.201 & $\begin{array}{c}1.07 \\
(0.94-1.22)\end{array}$ & 0.017 \\
\hline
\end{tabular}

${ }^{a}$ Number of comparisons.

${ }^{\mathrm{b}} P$-value of Q-test for heterogeneity test.

Table 3: Stratified analyses of the HOTAIR rs920778, PRNCR1 rs1016343, POLR2E rs3787016 on cancer risk

\begin{tabular}{|c|c|c|c|c|c|c|c|c|c|}
\hline \multirow{2}{*}{ Variables } & \multirow{2}{*}{$\mathbf{n}^{\mathbf{a}}$} & \multirow{2}{*}{ Cases } & \multirow{2}{*}{ Controls } & \multicolumn{2}{|c|}{ Dom model } & \multicolumn{2}{|c|}{ Rec model } & \multicolumn{2}{|c|}{ Add model } \\
\hline & & & & OR $(95 \%$ CI $)$ & $P^{b}$ & OR $(95 \%$ CI $)$ & $P^{b}$ & OR $(95 \%$ CI $)$ & $P^{b}$ \\
\hline $\begin{array}{l}\text { HOTAIR } \\
\text { rs920778 }\end{array}$ & 5 & 3627 & 4585 & $1.20(0.92-1.57)$ & 0.005 & $1.61(1.08-2.41)$ & $<0.001$ & $1.24(1.03-1.49)$ & 0.001 \\
\hline
\end{tabular}

Cancer type

\begin{tabular}{|c|c|c|c|c|c|c|c|c|c|}
\hline $\begin{array}{l}\text { Breast } \\
\text { cancer }\end{array}$ & 2 & 625 & 626 & $0.79(0.22-2.78)$ & 0.012 & $1.26(0.85-1.88)$ & 0.156 & $1.03(0.57-1.86)$ & 0.005 \\
\hline $\begin{array}{l}\text { Gastric } \\
\text { cancer }\end{array}$ & 2 & 904 & 1809 & $1.28(0.89-1.85)$ & 0.181 & $1.65(0.59-4.61)$ & 0.001 & $1.21(0.82-1.80)$ & 0.029 \\
\hline $\begin{array}{l}\text { Esophageal } \\
\text { cancer }\end{array}$ & 1 & 2098 & 2150 & $1.48(1.31-1.67)$ & - & $2.51(1.91-3.29)$ & - & $1.48(1.34-1.64)$ & - \\
\hline nicity & & & & & & & & & \\
\hline Asian & 3 & 3400 & 4254 & $1.46(1.33-1.61)$ & 0.953 & $2.13(1.42-3.20)$ & 0.004 & $1.46(1.35-1.57)$ & 0.803 \\
\hline Caucasian & 2 & 227 & 331 & $0.63(0.29-1.40)$ & 0.080 & $0.96(0.66-1.38)$ & 0.976 & $0.86(0.67-1.10)$ & 0.321 \\
\hline
\end{tabular}

(Continued) 


\begin{tabular}{|c|c|c|c|c|c|c|c|c|c|}
\hline \multirow{2}{*}{ Variables } & \multirow{2}{*}{$\mathbf{n}^{\mathrm{a}}$} & \multirow{2}{*}{ Cases } & \multirow{2}{*}{ Controls } & \multicolumn{2}{|c|}{ Dom model } & \multicolumn{2}{|c|}{ Rec model } & \multicolumn{2}{|c|}{ Add model } \\
\hline & & & & OR $(95 \%$ CI) & $P^{b}$ & OR $(95 \%$ CI) & $P^{b}$ & OR $(95 \%$ CI) & $P^{b}$ \\
\hline $\begin{array}{l}\text { PRNCR1 } \\
\text { rs1016343 }\end{array}$ & 7 & 3998 & 4284 & $1.27(1.04-1.53)$ & 0.002 & $1.33(0.92-1.92)$ & $<0.001$ & $1.24(1.04-1.47)$ & $<0.001$ \\
\hline \multicolumn{10}{|l|}{ Cancer Type } \\
\hline $\begin{array}{l}\text { Colorectal } \\
\text { cancer }\end{array}$ & 1 & 313 & 595 & $1.03(0.78-1.37)$ & - & $0.80(0.54-1.19)$ & - & $0.96(0.79-1.17)$ & - \\
\hline $\begin{array}{l}\text { Gastric } \\
\text { cancer }\end{array}$ & 1 & 219 & 394 & $1.00(0.71-1.41)$ & - & $0.69(0.44-1.09)$ & - & $0.90(0.71-1.14)$ & - \\
\hline $\begin{array}{l}\text { Prostate } \\
\text { cancer }\end{array}$ & 5 & 3466 & 3295 & $1.39(1.12-1.71)$ & 0.012 & $1.83(1.53-2.18)$ & 0.855 & $1.42(1.29-1.56)$ & 0.257 \\
\hline \multicolumn{10}{|l|}{ Ethnicity } \\
\hline $\begin{array}{l}\text { African } \\
\text { American }\end{array}$ & 1 & 143 & 79 & $0.78(0.45-1.36)$ & - & - & - & - & - \\
\hline Asian & 5 & 2602 & 2972 & $1.30(1.00-1.69)$ & 0.002 & $1.26(0.80-1.97)$ & $<0.001$ & $1.21(0.96-1.53)$ & $<0.001$ \\
\hline Caucasian & 1 & 1253 & 1233 & $1.39(1.18-1.63)$ & - & $1.72(1.21-2.44)$ & - & $1.36(1.19-1.55)$ & - \\
\hline $\begin{array}{l}\text { POLR2E } \\
\text { rs3787016 }\end{array}$ & 4 & 5841 & 6702 & $0.87(0.61-1.26)$ & 0.005 & $1.52(1.17-1.97)$ & 0.201 & $1.07(0.94-1.22)$ & 0.017 \\
\hline \multicolumn{10}{|l|}{ Cancer Type } \\
\hline $\begin{array}{l}\text { Esophageal } \\
\text { cancer }\end{array}$ & 1 & 369 & 370 & $0.73(0.53-0.99)$ & - & $1.76(1.25-2.49)$ & - & $0.97(0.79-1.19)$ & - \\
\hline $\begin{array}{l}\text { Prostate } \\
\text { cancer }\end{array}$ & 3 & 5472 & 6332 & $0.95(0.59-1.53)$ & 0.012 & $1.32(0.81-2.16)$ & 0.112 & $1.10(0.95-1.27)$ & 0.025 \\
\hline \multicolumn{10}{|l|}{ Ethnicity } \\
\hline Asian & 2 & 1384 & 1402 & $0.95(0.59-1.52)$ & 0.008 & $1.64(1.35-1.99)$ & 0.624 & $1.08(0.90-1.31)$ & 0.116 \\
\hline Caucasian & 2 & 4457 & 5300 & $0.73(0.52-1.02)$ & - & $0.94(0.51-1.72)$ & - & $1.01(0.70-1.44)$ & 0.007 \\
\hline
\end{tabular}

a Number of comparisons.

${ }^{\mathrm{b}} P$-value of Q-test for heterogeneity test.

SNPs (PRNCR1 rs1016343: $t=-1.16, P=0.311 ; P O L R 2 E$ rs3787016: $t=-3.20, P=0.085$ ) (Figure 2). Begg's funnle plot also showed the asymmetry for the HOTAIR rs920778, suggesting the existence of publication bias (Figure 2A). Furthermore, we used a trim-and-fill method to adjust the bias, which was developed by Duval and Tweedie [35]. The adjusted result from the random model was ORs of $1.26(1.06-1.46)$ for HOTAIR rs920778 in the additive model, which was similar with our results $(\mathrm{OR}=$ $1.24,95 \% \mathrm{CI}=1.03-1.49$ ).

\section{DISCUSSION}

LncRNAs participate in the diverse biological process, and abnormal expression of lncRNAs is associated with human cancers [36, 37]. Cumulative studies have suggested that IncRNAs polymorhisms have been widely studied, and are associated with cancer risk. However, results are not consistent. Thus, it is warranted to identify the association between lncRNAs polymorphisms and cancer risk. Through searching the PubMed, we screen the all published articles, and finally, we include the IncRNA HOTAIR, PRNCR1, H19, and POLR2E polymorphisms in this meta-analysis. The results indicate that these four lncRNAs polymorphisms (HOTAIR rs920778, PRNCR1 rs1016343 and rs16901946, POLR2E rs3787016) can contribute to the increased risk of cancer.

HOTAIR can bind to the polycomb-repressive complex 2 [5] and lysine specific demethylase 1 complex [38] to play the roles in cell biological process. At present, HOTAIR polymorphisms are the most commonly studied than other lncRNAs in human diseases, particularly in cancer. Interestingly, in recent two years, there have nine studies to investigate the association between HOTAIR polymorphisms and cancer risk. The polymorphisms in 
Table 4: Meta-regression analysis of the HOTAIR rs920778, PRNCR1 rs1016343, POLR2E rs3787016 on cancer risk (additive model)

\begin{tabular}{llcccc}
\hline SNPs & Variables & Coefficient & Standard error & $\boldsymbol{P}$ & $\mathbf{9 5 \%}$ CI \\
\hline HOTAIR rs920778 & $\begin{array}{l}\text { Ethnicity } \\
\text { Source of }\end{array}$ & 0.529 & 0.131 & $<0.001$ & $0.27-0.79$ \\
& $\begin{array}{l}\text { control } \\
\text { Cancer type }\end{array}$ & 0.168 & 0.349 & 0.631 & $-0.52-0.85$ \\
& $\begin{array}{l}\text { Genotyping } \\
\text { method }\end{array}$ & 0.529 & 0.181 & 0.672 & $-0.28-0.43$ \\
& $\begin{array}{l}\text { Ethnicity } \\
\text { Source of }\end{array}$ & 0.117 & 0.272 & 0.667 & $-0.42-0.65$ \\
\hline $\begin{array}{l}\text { control } \\
\text { Cancer type }\end{array}$ & 0.117 & 0.272 & 0.667 & $-0.42-0.65$ \\
& $\begin{array}{l}\text { Genotyping } \\
\text { method }\end{array}$ & 0.263 & 0.063 & $<0.001$ & $0.14-0.39$ \\
\hline POLR2E rs3787016343 & $\begin{array}{l}\text { Ethnicity } \\
\text { Source of } \\
\text { control }\end{array}$ & -0.053 & 0.202 & 0.795 & $-0.01-0.20$ \\
\hline $\begin{array}{l}\text { Cancer type } \\
\text { Genotyping }\end{array}$ & 0.265 & 0.087 & 0.002 & $0.09-0.45$ \\
method & 0.107 & 0.219 & 0.627 & $-0.32-0.54$ \\
& 0.092 & 0.046 & 0.047 & $0.00-0.18$ \\
\hline
\end{tabular}

HOTAIR will be a hot concern in predicting cancer risk. For example, Yan et al. and his colleagues proposed that HOTAIR rs920778 improved the risk of breast cancer in Chinese populations [14]. In the esophageal cancer, authors also observed the similar result [20]. However, in a Turkish population, no significant association was found in gastric cancer [17]. Our meta-analysis results indicated that rs920778 can increase the cancer susceptibility. In the subgroup analysis, we also observed the significant improved risk in Asians and in esophageal cancer. Further, we performed the combination analysis of HaploReg and RegulomeDB to annotate the function of HOTAIR rs920778. As shown in Supplementary Table 2, we found that rs920778 were related to DNase I hypersensitivity and DNA-binding motifs. For Asians, the $\mathrm{C}$ allele frequency was 0.76 , which was higher than in Caucasians (European) (0.69). Difference in genetic background may reflect the results. Herein, only one included study was involved in esophageal cancer [20], thus, we should interpret the findings with caution. Other common SNPs in HOTAIR (rs1899663 and rs4759314) also had been included in this meta-analysis, and no significant association is present.

Besides, we also investigated the effect of lncRNA PRNCR1 on cancer risk. PRNCR1 also known as PCAT8, was highly expressed in prostate cancer, and can mediate the prostate cancer cells gene activation programs and proliferation by binding to the androgen receptor [39].
Chung et al. used the resequencing and fine mapping of 8q24 region (Chr8: 128.14-128.28 Mb) and confirmed the PRNCR1 polymorphisms associated with prostate cancer risk [24]. Meanwhile, genetic variations in PRNCR1 also had been investigated in gastric cancer [22] and colorectal cancer [23]. In order to comprehensively evaluate the precise effect of PRNCRI rs1016343 on cancer risk, we performed a meta-analysis to summarize all published studies. Results indicated that rs1016343 indeed can predict the cancer susceptibility, particularly in prostate cancer and Caucasians. It was worth to note that the published studies were mainly involved in prostate cancer and Asians, thus, other cancer types and ethnicity should be further investigated to validate these findings. Functional annotation suggested rs1016343 exhibiting DNase I hypersensitivity, Protein binding and DNA-binding motifs (Supplementary Table 2), which may be related with PRNCR1 expression. Herein, we also evaluated the association of PRNCR1 rs13252298, rs7007694, rs16901946 and rs1456315 and cancer risk in this meta-analysis. The pooled results suggested that PRNCR1 rs16901946 was associated with increased risk of cancer, which was consistent with Chung et al. findings in prostate cancer [24]. We noticed that there were three studies regarding with rs 16901946 . However, rs 16901946 was not associated with gastric cancer and colorectal cancer risk [22-24]. In the future, more studies should be 
conducted to evaluate the effect of rs16901946 on cancer risk, such as prostate cancer, gastric cancer, colorectal cancer, etc.

Comparing with the above two lncRNA genes, POLR2E polymorphism was relatively less studied in cancer. So far, there are only four studies to investigate the association between POLR2E rs3787016 and cancer risk. Jin et al firstly reported the rs3787016 in $P O L R 2 E$ gene that was associated with prostate cancer susceptibility based on prostate cancer genome wide association study [7]. Subsequently, authors investigated the role of rs3787016 in esophageal cancer [33] and prostate cancer $[8,34]$. Meta-analysis results revealed that rs3787016 can predict the cancer risk. In the stratification analysis of cancer type, we observed that rs3787016 was associated with the risk of esophageal cancer, but not prostate cancer. We found that among the included four studies, three studies investigated the association between rs3787016 and prostate cancer risk [7, 8, 34], however, only Jin et al. identified the significant SNP rs3787016 with prostate cancer risk [7]. In addition, we observed that the population of included studies had both

A

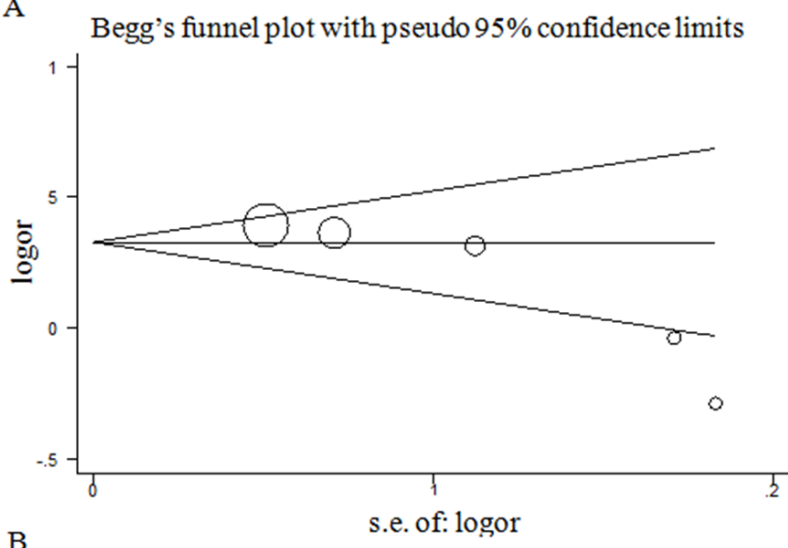

Begg's funnel plot with pseudo $95 \%$ confidence limits

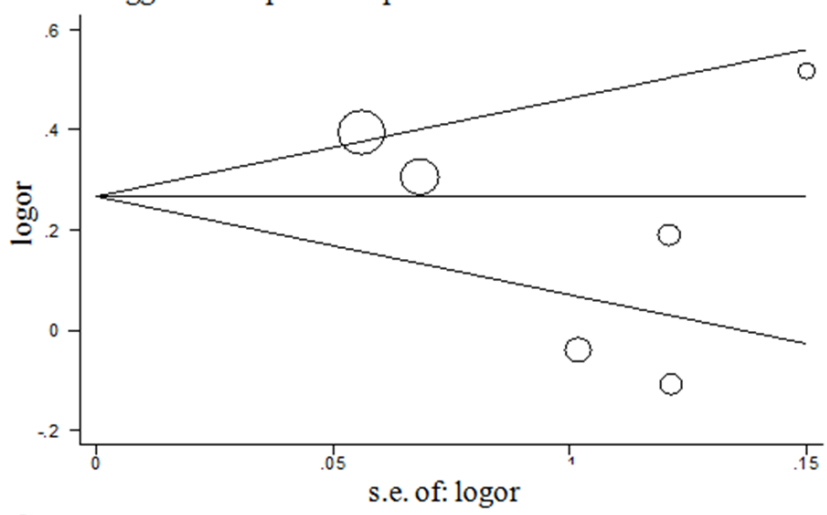

$\mathrm{C}$

Begg's funnel plot with pseudo $95 \%$ confidence limits

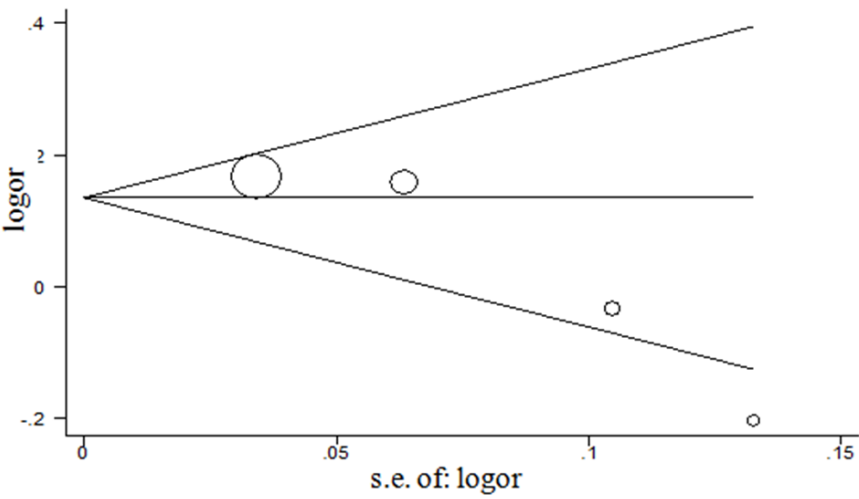

Figure 2: Begg's funnel plot for publication bias test (additive model). Each point represents a separate study for the indicated association. $\log [$ or], natural logarithm of OR. Horizontal line, mean effect size. A. HOTAIR rs920778. B. PRNCR1 rs1016343. C. POLR2E rs3787016. 
Asian and Caucasian populations. In the stratification analysis of ethnicity, there was significant association between rs3787016 and cancer risk in Asian population. Differences in genetic background may be a possible reflection of rs3787016 on cancer risk. Therefore, larger studies were warranted to be performed in prostate cancer and different ethnicity.

We also summarized the results of the lncRNA H19 polymorphisms with cancer risk. As early as 1990, Brannan et al. had identified the function of $\mathrm{H} 19$ gene as an RNA [40]. Aberrant expression of H19 had participated in multiple cancers $[41,42]$. There were many studies to investigate the SNPs within H19 associated with cancer susceptibility [27-32]. Bhatti et al. found that $H 19$ rs2107425 was not associated with overall breast cancer risk, however, individuals with the $H 19$ rs 2107425 variant alleles had the decreased risk of breast cancer in occupational radiation low-dose group and with the increased breast cancer risk in high-dose group [30]. In another population-based prospective cohort, we observed that rs2107425 variant alleles were associated with decreased risk of breast cancer [31]. Additionally, the effect of H19 rs2107425 also was investigated on the risk of bladder cancer and ovarian cancer. In bladder cancer, authors found the borderline effect for rs2107425 polymorphism [29], while in ovarian cancer, we did not observe the significant effect for rs 2107425 polymorphism [32]. Therefore, we performed a metaanalysis to summarize the results of rs2107425 and cancer risk. The pooled results showed that $H 19$ rs2107425 had the borderline effect on cancer risk. Further studies should validate our findings.

Due to the identification of large number of IncRNAs, aberrant expression of lncRNAs have been widely studied, and several meta-analysis have been done to evaluate the expression of IncRNAs and the prognosis of cancer [36, 43]. At present, there have no study to systematically investigate the association between lncRNAs polymorphisms and cancer risk. Herein, we focused on exploring the common lncRNAs polymorphisms associated with cancer risk in a metaanalysis. Interestingly, we indeed found the lncRNAs polymorphisms were related with cancer risk. These findings can provide the useful information to support the further study in the function of lncRNAs polymorphisms. However, we also noticed that among the included studies were shown the significant heterogeneity. Meta-regression revealed that the source of heterogeneity mainly from the ethnicity, genotyping method, cancer type, and source of control, suggesting these four factors playing the crucial roles.

Although we observed the significant association between lncRNAs polymorphisms and cancer risk in this meta-analyais, however, several limitations should be warranted. Due to lacking the detailed data of included studies, our evaluation mainly focused on unadjusted results of lncRNAs polymorphisms and cancer risk. Additionally, our meta-analysis included several small sample size studies, which may influence the results stability. It was worth to note that the majority of studies matching with age, sex and residential environment can control the selection bias.

In conclusion, our meta-analysis showed that lncRNA HOTAIR rs920778, PRNCR1 rs1016343 and rs16901946, POLR2E rs3787016 were associated with cancer susceptibility. However, due to several confounding factors, we should explain the results with caution. Further larger and multi-ethnicity studies should confirm our findings. Moreover, genetic factors should combine with environmental factors to predict the risk of cancer, which will lead to comprehensively understand the association between lncRNAs polymorphisms and cancer risk.

\section{MATERIALS AND METHODS}

\section{Study selection}

We performed a comprehensive article search in PubMed up to February 2016 using the key words 'IncRNA' or 'long non-coding RNA' and 'polymorphism' to summarize the articles of relevant lncRNA polymorphisms and cancer risk. Besides, we also used the manual search of the references of relavant articles. The included studies should meet some criteria: firstly, studies should be written in English language; secondly, studies should be a human case-control design; thirdly, studies had to report the genotypes frequency. We also had some excluded criteria: firstly, only one or two studies investigated one particular lncRNA gene, which was not suitable for meta-analysis; secondly, two studies used the same data, and we chose the study with largest sample size.

\section{Data extraction}

Two authors (Chu $\mathrm{H}$ and Chen Y) independently extracted the available data from the selected studies, and data should be crosschecked. We mainly focused on collecting the following data: first author's name, year of publication, country, ethnicity, source of control (hospitalbased or population-based), genotyping method, genotype frequency of SNPs, case and control numbers, matching factors, Hardy-Weingberg equilibrium (HWE) and cancer type. We categorized ethnicity as Asian, Caucasian, or African decent population. If one study was composed of multiple ethnic populations and we can not distinguish the different ethnicity among the multiple ethnic populations, we named it mixed population [30]. For study including Caucasian and African decent population (Salinas et al., 2008), we separately extracted the data for each ethnic 
group [6]. There were three studies without data for all three genotypes, therefore, we calculated the odds ratios (ORs) for dominant model [6,30] and additive model [7] in statistical analysis.

\section{Statistical analysis}

In this meta-analysis, we mainly used the ORs and $95 \%$ confidence intervals (CIs) to investigate the association between lncRNA polymorphism and cancer risk. For lncRNA polymorphisms, we calculated the risks of the dominant model, recessive model and additive model (linearly according to $0,1,2$ minor allele). For example, $\mathrm{A}$ is the wild allele, and $\mathrm{B}$ is the variant allele. In the dominant model, we estimated the risk of the variant genotype $\mathrm{AB}$ and $\mathrm{BB}$, compared with wild genotype $\mathrm{AA}$ $(\mathrm{AB} / \mathrm{BB}$ versus $\mathrm{AA})$, and evaluated the risk of $\mathrm{BB}$ versus $A B / A A$, assuming recessive effects of the variant $B$ allele. It should be mentioned that there were two studies without data for all three genotypes $[6,7]$, therefore, we calculated the results in recessive model and additive model, respectively. In addition, we also performed the stratified analyses of ethnicity and cancer type.

The meta-analysis of included studies was conducted with a random-effect model (the DerSimonian and Laird method), which mainly considered the variability within or between study [44]. The Cochran's Q-test and I ${ }^{2}$ were used for assessment of studies heterogeneity, and heterogeneity was supposed to be significant when $P$ value was less than 0.10 [45]. In this study, we further examined the ethnicity, source of control and genotyping methods in metaregression model to explore the source of heterogeneity. The Tau-squared was used to estimate of between study variance and the less $P_{\text {heterogeneity }}$ value was, the more tausquared was [46]. We always used the Begg's and Egger's test to assess the publication bias of included studies. Publication bias was shown, when $P$ value was less than 0.05. HaploReg (v4.1) and RegulomeDB (v1.1) were used to annotate the function of SNPs.

All statistical analyses were conducted with the STATA 12.0 (StataCorp, College Station, TX). A $P$ value $<$ 0.05 was considered significantly.

\section{ACKNOWLEDGMENTS}

This study was partly supported by National Natural Science Foundation of China (81502866, 81473050), Jiangsu Provincial Postdoctoral Science Foundation funded project (1501081C), China Postdoctoral Science Foundation funded project (2015M580449), Collaborative Innovation Center For Cancer Personalized Medicine, and the Priority Academic Program Development of Jiangsu Higher Education Institutions (Public Health and Preventive Medicine). The funders had no role in study design, data collection and analysis, decision to publish, or preparation of the manuscript.

\section{CONFLICTS OF INTEREST}

We declared no conflicts of interest in this study.

\section{REFERENCES}

1. Nagano T, Mitchell JA, Sanz LA, Pauler FM, FergusonSmith AC, Feil R, Fraser P. The Air noncoding RNA epigenetically silences transcription by targeting G9a to chromatin. Science. 2008; 322:1717-1720.

2. Hung T, Wang Y, Lin MF, Koegel AK, Kotake Y, Grant GD, Horlings HM, Shah N, Umbricht C, Wang P, Wang Y, Kong B, Langerod A, et al. Extensive and coordinated transcription of noncoding RNAs within cell-cycle promoters. Nat Genet. 2011; 43:621-629.

3. Derrien T, Johnson R, Bussotti G, Tanzer A, Djebali S, Tilgner H, Guernec G, Martin D, Merkel A, Knowles DG, Lagarde J, Veeravalli L, Ruan X, et al. The GENCODE v7 catalog of human long noncoding RNAs: analysis of their gene structure, evolution, and expression. Genome Res. 2012; 22:1775-1789.

4. Ponting CP, Oliver PL, Reik W. Evolution and functions of long noncoding RNAs. Cell. 2009; 136:629-641.

5. Gupta RA, Shah N, Wang KC, Kim J, Horlings HM, Wong DJ, Tsai MC, Hung T, Argani P, Rinn JL, Wang Y, Brzoska P, Kong B, et al. Long non-coding RNA HOTAIR reprograms chromatin state to promote cancer metastasis. Nature. 2010; 464:1071-1076.

6. Salinas CA, Kwon E, Carlson CS, Koopmeiners JS, Feng Z, Karyadi DM, Ostrander EA, Stanford JL. Multiple independent genetic variants in the $8 \mathrm{q} 24$ region are associated with prostate cancer risk. Cancer Epidemiol Biomarkers Prev. 2008; 17:1203-1213.

7. Jin G, Sun J, Isaacs SD, Wiley KE, Kim ST, Chu LW, Zhang Z, Zhao H, Zheng SL, Isaacs WB, Xu J. Human polymorphisms at long non-coding RNAs (lncRNAs) and association with prostate cancer risk. Carcinogenesis. 2011; 32:1655-1659.

8. Nikolic ZZ, Brajuskovic GN, Pavicevic D, Kojic AS, Vukotic VD, Tomovic SM, Cerovic SJ, Filipovic V, Misljenovic D, Romac SP. Assessment of possible association between rs3787016 and prostate cancer risk in Serbian population. Int J Clin Exp Med. 2013; 6:57-66.

9. Schmidt LH, Spieker T, Koschmieder S, Schaffers S, Humberg J, Jungen D, Bulk E, Hascher A, Wittmer D, Marra A, Hillejan L, Wiebe K, Berdel WE, et al. The long noncoding MALAT-1 RNA indicates a poor prognosis in non-small cell lung cancer and induces migration and tumor growth. J Thorac Oncol. 2011; 6:1984-1992.

10. Rinn JL, Kertesz M, Wang JK, Squazzo SL, Xu X, Brugmann SA, Goodnough LH, Helms JA, Farnham PJ, Segal E, Chang HY. Functional demarcation of active and silent chromatin domains in human HOX loci by noncoding RNAs. Cell. 2007; 129:1311-1323. 
11. Xue Y, Ma G, Zhang Z, Hua Q, Chu H, Tong N, Yuan L, Qin C, Yin C, Zhang Z, Wang M. A novel antisense long noncoding RNA regulates the expression of MDC1 in bladder cancer. Oncotarget. 2015; 6:484-493. doi: 10.18632/ oncotarget.2861.

12. Gaffney DJ. Global properties and functional complexity of human gene regulatory variation. PLoS Genet. 2013; 9:e1003501.

13. Bayram S, Sumbul AT, Dadas E. A functional HOTAIR rs12826786 C $>\mathrm{T}$ polymorphism is associated with breast cancer susceptibility and poor clinicopathological characteristics in a Turkish population: a hospital-based case-control study. Tumour Biol. 2016; 37:5577-5584.

14. Yan R, Cao J, Song C, Chen Y, Wu Z, Wang K, Dai L. Polymorphisms in IncRNA HOTAIR and susceptibility to breast cancer in a Chinese population. Cancer Epidemiol. 2015; 39:978-985.

15. Bayram S, Sumbul AT, Batmaci CY, Genc A. Effect of HOTAIR rs920778 polymorphism on breast cancer susceptibility and clinicopathologic features in a Turkish population. Tumour Biol. 2015; 36:3863-3870.

16. Du M, Wang W, Jin H, Wang Q, Ge Y, Lu J, Ma G, Chu H, Tong N, Zhu H, Wang M, Qiang F, Zhang Z. The association analysis of lncRNA HOTAIR genetic variants and gastric cancer risk in a Chinese population. Oncotarget. 2015; 6:31255-31262. doi: 10.18632/oncotarget.5158.

17. Bayram S, Ulger Y, Sumbul AT, Kaya BY, Rencuzogullari A, Genc A, Sevgiler Y, Bozkurt O, Rencuzogullari E. A functional HOTAIR rs920778 polymorphism does not contributes to gastric cancer in a Turkish population: a casecontrol study. Fam Cancer. 2015; 14:561-567.

18. Pan W, Liu L, Wei J, Ge Y, Zhang J, Chen H, Zhou L, Yuan Q, Zhou C, Yang M. A functional lncRNA HOTAIR genetic variant contributes to gastric cancer susceptibility. Mol Carcinog. 2016; 55:90-96.

19. Guo W, Dong Z, Bai Y, Guo Y, Shen S, Kuang G, Xu J. Associations between polymorphisms of HOTAIR and risk of gastric cardia adenocarcinoma in a population of north China. Tumour Biol. 2015; 36:2845-2854.

20. Zhang X, Zhou L, Fu G, Sun F, Shi J, Wei J, Lu C, Zhou C, Yuan Q, Yang M. The identification of an ESCC susceptibility SNP rs920778 that regulates the expression of IncRNA HOTAIR via a novel intronic enhancer. Carcinogenesis. 2014; 35:2062-2067.

21. Xue Y, Gu D, Ma G, Zhu L, Hua Q, Chu H, Tong N, Chen J, Zhang Z, Wang M. Genetic variants in lncRNA HOTAIR are associated with risk of colorectal cancer. Mutagenesis. 2015; 30:303-310.

22. Li L, Jia F, Bai P, Liang Y, Sun R, Yuan F, Zhang L, Gao L. Association between polymorphisms in long non-coding RNA PRNCR1 in 8q24 and risk of gastric cancer. Tumour Biol. 2016; 37:299-303.

23. Li L, Sun R, Liang Y, Pan X, Li Z, Bai P, Zeng X, Zhang D, Zhang L, Gao L. Association between polymorphisms in long non-coding RNA PRNCR1 in 8q24 and risk of colorectal cancer. J Exp Clin Cancer Res. 2013; 32:104.

24. Chung S, Nakagawa H, Uemura M, Piao L, Ashikawa K, Hosono N, Takata R, Akamatsu S, Kawaguchi T, Morizono T, Tsunoda T, Daigo Y, Matsuda K, et al. Association of a novel long non-coding RNA in 8q24 with prostate cancer susceptibility. Cancer Sci. 2011; 102:245-252.

25. Hui J, Xu Y, Yang K, Liu M, Wei D, Wei D, Zhang Y, Shi XH, Yang F, Wang N, Zhang Y, Wang X, Liang S, et al. Study of genetic variants of 8q21 and 8q24 associated with prostate cancer in Jing-Jin residents in northern China. Clin Lab. 2014; 60:645-652.

26. Zheng SL, Hsing AW, Sun J, Chu LW, Yu K, Li G, Gao Z, Kim ST, Isaacs WB, Shen MC, Gao YT, Hoover RN, $\mathrm{Xu}$ J. Association of 17 prostate cancer susceptibility loci with prostate cancer risk in Chinese men. Prostate. 2010; 70:425-432.

27. Yang $\mathrm{C}$, Tang $\mathrm{R}$, Ma $\mathrm{X}$, Wang $\mathrm{Y}$, Luo $\mathrm{D}, \mathrm{Xu} \mathrm{Z}$, Zhu Y, Yang L. Tag SNPs in long non-coding RNA H19 contribute to susceptibility to gastric cancer in the Chinese Han population. Oncotarget. 2015; 6:15311-15320. doi: 10.18632/oncotarget.3840.

28. Soares MR, Huber J, Rios AF, Ramos ES. Investigation of IGF2/ApaI and H19/RsaI polymorphisms in patients with cutaneous melanoma. Growth Horm IGF Res. 2010; 20:295-297.

29. Verhaegh GW, Verkleij L, Vermeulen SH, den Heijer M, Witjes JA, Kiemeney LA. Polymorphisms in the H19 gene and the risk of bladder cancer. Eur Urol. 2008; 54:1118-1126.

30. Bhatti P, Doody MM, Alexander BH, Yuenger J, Simon SL, Weinstock RM, Rosenstein M, Stovall M, Abend M, Preston DL, Pharoah P, Struewing JP, Sigurdson AJ. Breast cancer risk polymorphisms and interaction with ionizing radiation among U.S. radiologic technologists. Cancer Epidemiol Biomarkers Prev. 2008; 17:2007-2011.

31. Butt S, Harlid S, Borgquist S, Ivarsson M, Landberg G, Dillner J, Carlson J, Manjer J. Genetic predisposition, parity, age at first childbirth and risk for breast cancer. BMC Res Notes. 2012; 5:414.

32. Song H, Ramus SJ, Kjaer SK, DiCioccio RA, ChenevixTrench G, Pearce CL, Hogdall E, Whittemore AS, McGuire V, Hogdall C, Blaakaer J, Wu AH, Van Den Berg DJ, et al. Association between invasive ovarian cancer susceptibility and 11 best candidate SNPs from breast cancer genome-wide association study. Hum Mol Genet. 2009; 18:2297-2304.

33. Kang M, Sang Y, Gu H, Zheng L, Wang L, Liu C, Shi Y, Shao A, Ding G, Chen S, Tang W, Yin J. Long noncoding RNAs POLR2E rs3787016 C/T and HULC rs7763881 $\mathrm{A} / \mathrm{C}$ polymorphisms are associated with decreased risk of esophageal cancer. Tumour Biol. 2015; 36:6401-6408.

34. Cao DL, Gu CY, Zhu Y, Dai B, Zhang HL, Shi GH, Shen YJ, Zhu YP, Ma CG, Xiao WJ, Qin XJ, Lin GW, Ye DW. 
Polymorphisms at long non-coding RNAs and prostate cancer risk in an eastern Chinese population. Prostate Cancer Prostatic Dis. 2014; 17:315-319.

35. Duval S, Tweedie R. Trim and fill: a simple funnel-plotbased method of testing and adjusting for publication bias in meta-analysis. Biometrics. 2000; 56:455-463.

36. Ma G, Wang Q, Lv C, Qiang F, Hua Q, Chu H, Du M, Tong N, Jiang Y, Wang M, Zhang Z, Wang J, Gong W. The prognostic significance of HOTAIR for predicting clinical outcome in patients with digestive system tumors. J Cancer Res Clin Oncol. 2015; 141:2139-2145.

37. Liu YR, Jiang YZ, Xu XE, Hu X, Yu KD, Shao ZM. Comprehensive transcriptome profiling reveals multigene signatures in triple-negative breast cancer. Clin Cancer Res. 2016; 22:1653-1662.

38. Tsai MC, Manor O, Wan Y, Mosammaparast N, Wang JK, Lan F, Shi Y, Segal E, Chang HY. Long noncoding RNA as modular scaffold of histone modification complexes. Science. 2010; 329:689-693.

39. Yang L, Lin C, Jin C, Yang JC, Tanasa B, Li W, Merkurjev D, Ohgi KA, Meng D, Zhang J, Evans CP, Rosenfeld MG. IncRNA-dependent mechanisms of androgen-receptor-regulated gene activation programs. Nature. 2013; 500:598-602.

40. Brannan CI, Dees EC, Ingram RS, Tilghman SM. The product of the $\mathrm{H} 19$ gene may function as an RNA. Mol Cell Biol. 1990; 10:28-36.

41. Medrzycki M, Zhang Y, Zhang W, Cao K, Pan C, Lailler N, McDonald JF, Bouhassira EE, Fan Y. Histone h1.3 suppresses h19 noncoding RNA expression and cell growth of ovarian cancer cells. Cancer Res. 2014; 74:6463-6473.

42. Zhu M, Chen Q, Liu X, Sun Q, Zhao X, Deng R, Wang Y, Huang J, Xu M, Yan J, Yu J. lncRNA H19/miR-675 axis represses prostate cancer metastasis by targeting TGFBI. FEBS J. 2014; 281:3766-3775.

43. Tian $\mathrm{X}, \mathrm{Xu}$ G. Clinical value of lncRNA MALAT1 as a prognostic marker in human cancer: systematic review and meta-analysis. BMJ Open. 2015; 5:e008653.

44. DerSimonian R, Laird N. Meta-analysis in clinical trials. Control Clin Trials. 1986; 7:177-188.

45. Lau J, Ioannidis JP, Schmid CH. Quantitative synthesis in systematic reviews. Ann Intern Med. 1997; 127:820-826.

46. Whitehead A, Whitehead J. A general parametric approach to the meta-analysis of randomized clinical trials. Stat Med. $1991 ; 10: 1665-1677$. 